\title{
Sequential letter and word recognition in deaf and hearing subjects*
}

\author{
RICHARD D. ZAKIA $†$ and RALPH NORMAN HABER \\ University of Rochester, Rochester, New York 14627
}

To examine the processing of sequentially presented letters of familiar and nonsense words, especially among Ss of vastly differing experience on sequential tasks, three groups of Ss were tested on letters of words spelled sequentially on an alphanumeric display and on letters of words fingerspelled. These were a deaf group $(\mathrm{N}=33)$ with little or no hearing and who varied in their fingerspelling ability; a staff group $(\mathrm{N}=12)$ who taught fingerspelling and were highly proficient; and a hearing group $(\mathrm{N}=19)$. Of principal interest was the finding that the hearing Ss did better on nonsense letter recognition, while the deaf group did better on word recognition. Word length was important except to the staff Ss on fingerspelled words, which also suggests that concentration on fingerspelling proficiency forces attention to the whole word and not its component letters. Hearing Ss, who are the group faced with an unfamiliar task, seemed to attend to each letter and hence had more difficulty with recognition of the longer unit.

Many deaf persons depend on fingerspelling as one form of manual communication. The American Manual Alphabet is a system which describes a variety of finger configurations, each representing one of the 26 letters and 10 numbers. For example, to fingerspell the word "love," a person would use four different finger configurations, each representing a letter (see Fig. 1). He would form these configurations in a rapid sequence, one after the other, each requiring about one-fifth of a second (Bomstein, 1965).

Perception of fingerspelled words is related to sequential tachistoscopic tasks such as those used by Haber and Nathanson (1969) and Kolers and Katzman (1966). Each symbol is briefly presented in sequence in the same spatial location. Although there are some similarities, with fingerspelling the symbols are in motion. They are not presented discretely as are printed letters in a tachistoscopic task. Further, the symbols are sent with the expressive backgrounds of the sender (smiles, frowns, pantomime) as additional cues to recognition.

One purpose of this study was to examine, with deaf Ss whose fingerspelling experience and ability vary, whether or not that variation was related to their

*This research was partially supported by grants from the National Science Foundation, GB 4547 and GB 5910, and from the United States Public Health Service, MH 10753, to the second author. We would like to gratefully acknowledge the assistance of the staff of the National Technical Institute for the Deaf in this project. Part of this study was submitted in fulfillment of the doctoral degree at the University of Rochester for the first author.

How at the Rochester Institute of Technology, Instructional Research and Development, Rochester, New York 14623. proficiency on a sequential tachistoscopic recognition task used by Haber and Nathanson (1969). Since a partial or total deficit in hearing implies a marked reduction in sequential perceptual experience (audition being a uniquely sequential sensory system), one might question if deaf Ss may be handicapped on sequential tasks due to lack of experience rather than from deafness itself. Therefore, comparing deaf Ss on their performance in reading fingerspelled letters and words with that of reading tachistoscopically presented letters and words should provide some answers to this question.

A second purpose was to compare deaf and hearing Ss on the tachistoscopic task, using sequences of letters composing nonsense as well as familiar words. Of particular interest was the question of the way the two groups of Ss attend to the individual letters when they are constructing words. Hearing Ss, who are relatively inexperienced in sequential single-letter visual tasks might be expected to have to attend to the letters more and hence would be better at nonsense as compared to word stimuli, at least as compared to deaf Ss. Given the great current interest in whether meaningful materials are perceived without first having to recognize all of their component parts, this analysis seemed appropriate.

\section{Subjects \\ Three groups of volunteer Ss were} tested: deaf -33 students with hearing impairments enrolled at the Rochester Institute of Technology and being provided educational support service by the National Technical Institute for the Deaf at R.I.T.; hearing-19 students at R.I.T. with no hearing impairment; and staff-12 staff members at N.T.I.D., 6 deaf and 6 hearing, all highly proficient in fingerspelling.

The deaf and hearing students were enrolled in a variety of the colleges and departments at R.I.T. The deaf students had various levels of hearing impairment ranging from partial hearing in one ear (with a hearing aid) to no hearing in either ear. In addition, their primary modes of communication varied-some were speechreaders, some fingerspellers. Many of the latter also used sign language. None of the Ss in any group had participated in a psychophysical experiment previously.

\section{Apparatus}

For the tachistoscopic presentations the apparatus was the same as that used by Haber and Nathanson (1969). The letters were presented on a single alphanumeric display drawn on a $4 \times 3$ in. electroluminescent panel made by Massey Dickinson. The panel contained 15 segments, each of which could be controlled and displayed independently of the others by a PDP-8 digital computer. A 15-bit buffer served as interface between the accumulator of the PDP-8 and the segments on the panel. The program specified for each trial the time that the segments for each letter were on, the time between successive letters, and the particular sequence of letters to be displayed.

All letters were capitals, averaging $3 \frac{1}{1} \mathrm{in}$. high and 2 in. wide $(8 \times 5 \mathrm{deg}$ at a distance of $2 \mathrm{ft}$-see Fig. 2). The luminance of each segment was $8 \mathrm{fL}$. A weak luminance (less than $0.5 \mathrm{fL}$ ) was present, owing to stray light on the background of the panel when some segments were on. They were invisible when off. The room was dimly illuminated to allow the Ss to write their responses. A $25-\mathrm{W}$ lamp $3 \mathrm{ft}$ behind the $S$ and shielded from his view provided an illuminance of $1.3 \mathrm{~lm} / \mathrm{m}^{2}$ in the area in which the Ss were seated.

For the fingerspelled presentations the letters were presented to from one to five Ss at a time in a well-illuminated classroom. Examples of the fingerspelled

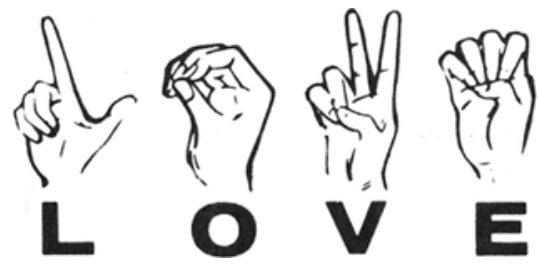

Fig. 1. A sequence of finger configurations in the American Manual Alphabet that represent the letters of the word "Iove." 
Table 1

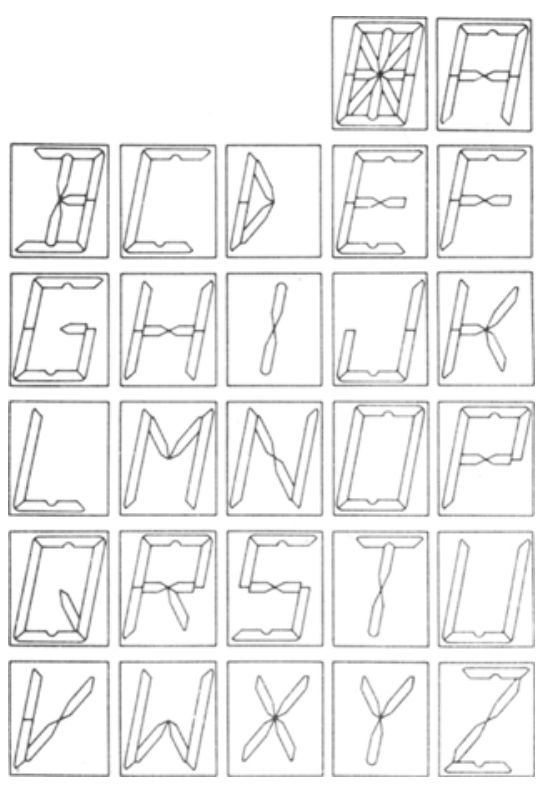

Fig. 2. Style of letters constructed from segments of the alphanumeric display used in the experiment.

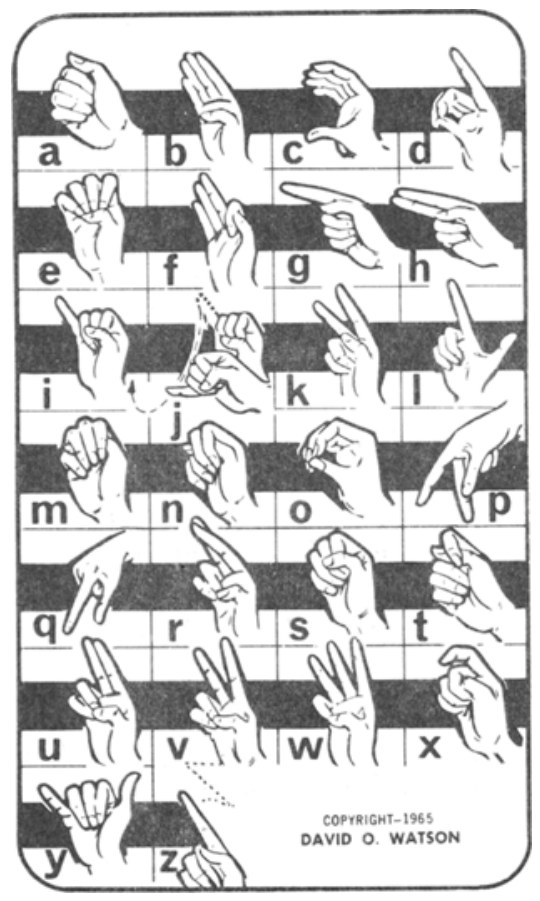

Fig. 3. Fingerspelled letters of the American Manual Alphabet.

letters are shown in Fig. 3. They were sent by a deaf person judged excellent in the clarity and consistency of his fingerspelling ability. The judging was done by seven N.T.I.D. staff members highly qualified in fingerspelling.

The sender was told to "send at a constant rate and make each letter
Words Presented by Computer and by Fingerspelling

Computerspelled Words at Three Different Rates

\begin{tabular}{|c|c|c|c|c|c|}
\hline \multicolumn{3}{|c|}{ Low Imagery } & \multicolumn{3}{|c|}{ High Imagery } \\
\hline 300 & 150 & 75 & 300 & 150 & 75 \\
\hline SOUL. & IDEA & FACT & GIRL & FROG & FIRE \\
\hline MIND & FATE & HINT & SHIP & GOLF & STAR \\
\hline DUTY & PACT & HOPE & LAKE & WINE & MEAT \\
\hline $\operatorname{COST}$ & LIFE & HOUR & FLAG & MULE & FORK \\
\hline HIDE & JOKE & TIME & NAIL & WIFE & LAWN \\
\hline STUB & IORM & CODE & OVLN & SKIN & PIPE \\
\hline SURTAX & ORIGIN & CHANCE & GARDEN & HAMMER & VALLEY \\
\hline EXCUSE & RATING & MOMENT & FOREST & FLOWER & CHURCH \\
\hline THEORY & ANSWER & METHOD & MOTHER & FRIEND & DOCTOR \\
\hline MEMORY & ADVICE & SAVANT & WINTER & SQUARE & CATTLE \\
\hline PLEDGE & EFIORT & CUSTOM & PENCIL & INFANT & HURDLE \\
\hline LENGTH & VIRTUE & SPIRIT & ROBBER & CIRCLE & CRADLE \\
\hline TENDENCY & ATTITUDE: & REMINDER & ELEPHANT & MOUNTAIN & REVOLVER \\
\hline INTEREST & NONSENSE & POSITION & UMBRELLA & MOSQUITO & MAGAZINE \\
\hline NAMESAKE & JEOPARDY & EVIDENCE & HOSPITAL & MACARONI & DAYBREAK \\
\hline CAPACITY & HLREDT TY & QUANTITY & PRISONER & RAILROAD & LEMONADE \\
\hline MAJORITY & CONTENTS & SEMESTER & CLOTHING & MUSICINN & MARRIAGE \\
\hline VELOCITY & RLACTION & PRESTIGL & MOISTURE & GARMENTS & INDUSTRY' \\
\hline \multicolumn{6}{|c|}{ Fingerspelled Words } \\
\hline MOOD & PATENT & OCCASION & BIRD & BREAST & BUILDING \\
\hline LINK & UPKEEP & HARDSHIP & GOLD & STREET & TWEEZERS \\
\hline CORE & DECEIT & PRESSURI & JAIL & DOLLAR & HHLLSIDE \\
\hline ODOR & (RISIS & CITATION & CORN & BEAVER & FOOTWEAR \\
\hline LICE & MENACL & ATROCITY & lORK & PRISON & BEVERAGE \\
\hline JURY & HATRED & VOCATION & COIN & STRING & DAYLIGHT \\
\hline LIME: & VANITY & CONOUEST & $\mathrm{HOME}$ & POSTER & BASEMENT \\
\hline DEED & HEALTH & IRICTION & TANK & TICKET & LEGGINGS \\
\hline LUMP & ERRANI) & MISCHIEI: & ROC' & MARKET & LECTURER \\
\hline BARD & SAIETY & EXIERIION & ARMY & SINGER & KERCHIEI: \\
\hline
\end{tabular}

distinctly. After a word or a group of the sender's difficulty in sending the letters has been sent, slowly drop your hand. Bring your hand back up to position before beginning the next word. Wait until everyone has written the word and is looking at you before sending the next word. (The timc between words is not important.) If you make a mistake in sending a word. stop, let everyone know it was a mistake, and then start the word over again. Begin each session with about a 5 -min practice session. First, send the alphabel a tew times. This will allow the sender and receiver to adjust to each other. Avoid facial cxpressions or any other clue that might help the reader identify the word or letters being sent."

Measures wore takell of the sending rate of fingerspelled letters of words and nonsense. These averaged 485 and $527 \mathrm{msec}$ per letter, respectively, for the deaf Ss (standard deviations of 22 and $26 \mathrm{msec}$ per letter. respectively) and 162 and 190 (with SDs of 21 and 16) for the staff Ss. The much slower rate used for the deaf Ss was necessary to allow the students with little experience in fingerspelling to demonstrate their ability. Some of the students did not know fingerspelling when they arrived at R.I.T., and their only experience with it was during the past school year. The longer sending rates used for the nonsense word task resulted from peculiar letter combinations. The usual rate for sending fingerspelled words in context to a proficient reader is about $200 \mathrm{msec}$ per letter.

\section{Stimuli}

Tables 1 and 2 contain the words and letters sent by computer and by fingerspelling, categorized by rate of presentation and imagery of words. The 4and 6-letter nonsense words (Table 2) were constructed by random selection from the distribution of the letters that made up the 4- and 6-letter words used in the word task. In some cases this resulted in a 3-or 4-letter word within the letter array. When this occurred, the letters within the array were again scrambled. Eight-letter arrays were not used because pilot tests showed that $S$ s had great difficulty giving a written response, even at the slowest rate. The imagery measure was taken from Paivio, Yuille, and Madigan (1968). Words were selected to be as equal as possible on imagery with each cell.

For each rate of sending, the computerspelled letters were displayed for $25 \mathrm{msec}$ per letter. To produce different rates, the off time between letters was set at 275,125 , and $50 \mathrm{msec}$ per letter, respectively. Words used during the practice sessions were selected from the 
Table 2

Letters Used in the Computerspelled and Fingerspelled Nonsense Words

\begin{tabular}{|c|c|c|}
\hline 300 & $\begin{array}{l}\text { erspelled } \\
150\end{array}$ & 75 \\
\hline $\begin{array}{l}\text { GHKG } \\
\text { IEFI } \\
\text { TSTI } \\
\text { YNCO } \\
\text { RLPL } \\
\text { LSNA } \\
\text { NVAI } \\
\text { USDO } \\
\text { TDUS } \\
\text { OAEL } \\
\text { HTDB } \\
\text { EISU }\end{array}$ & $\begin{array}{l}\text { AICE } \\
\text { DTLE } \\
\text { FMKO } \\
\text { WGFN } \\
\text { KSIE } \\
\text { ETPI } \\
\text { GLIO } \\
\text { OFWI } \\
\text { AFAO } \\
\text { LREU } \\
\text { EFNM } \\
\text { RJFE }\end{array}$ & $\begin{array}{l}\text { KTFA } \\
\text { EOTM } \\
\text { PNPA } \\
\text { IATF } \\
\text { CDEI } \\
\text { IRWE } \\
\text { ESER } \\
\text { TNHC } \\
\text { RLMO } \\
\text { AOEH } \\
\text { IPRF } \\
\text { HTOU }\end{array}$ \\
\hline $\begin{array}{l}\text { GTRTIO } \\
\text { OGHXSE } \\
\text { RURYPE } \\
\text { ARHLRE } \\
\text { UCORLE } \\
\text { EHXMDT } \\
\text { REERPO } \\
\text { AETEBG } \\
\text { OSYMLN } \\
\text { NFONEC } \\
\text { DSRBEW } \\
\text { ETMNIE }\end{array}$ & $\begin{array}{l}\text { NRNVOG } \\
\text { IATIFU } \\
\text { ESAIRO } \\
\text { AOERTC } \\
\text { HLIANI } \\
\text { MWNEIE } \\
\text { ERFQFC } \\
\text { MDSNRE } \\
\text { RFRLUA } \\
\text { IEDRVE } \\
\text { RTVCTI } \\
\text { GNEAFR }\end{array}$ & $\begin{array}{l}\text { EHCTLV } \\
\text { OTOTAO } \\
\text { HMNHSM } \\
\text { AEOTCP } \\
\text { NDNSUI } \\
\text { ATULEL } \\
\text { LRORHU } \\
\text { CTMASR } \\
\text { EMEVTI } \\
\text { LCRCEA } \\
\text { EHDRDA } \\
\text { YCOTDC }\end{array}$ \\
\hline
\end{tabular}

\begin{tabular}{lllll}
\multicolumn{3}{c}{ Fingerspelled } & Letters \\
\cline { 4 - 5 } \multicolumn{2}{c}{527} & & & 190 \\
\hline IMKD & URCEIR & & CFJO & EBEIRI \\
ORNG & PIOESD & & EOUI & TEHNSE \\
ODLO & AKTECE & & LMKE & VSLERT \\
DBIL & ETDTIA & & IEDT & NTGTRT \\
OLRC & PBERSA & MHDA & ENTMAI \\
EDRI & AVCNHS & EOEN & IRHNCA \\
OJRO & TNERLT & OCDY & TPERDK \\
LRCN & ERMPTR & UKBA & YEOKEA \\
IKYC & PDAOAG & MRAR & SFTSNE \\
RNAO & ELTSRS & PMLR & AEYIAR \\
\hline
\end{tabular}

same population of words used in the experiment and were used only in the practice sessions.

In general, in each condition the first few words were selected to be 4- and 6 -letter words at slow rates; the rest were ordered randomly over rate of presentation, imagery, and word length.

All deaf Ss were tested first with computerspelled words, then computerspelled nonsense words, then fingerspelled words, and then fingerspelled nonsense words. The hearing Ss received only the two computerspelled conditions, while the staff Ss, only the two fingerspelled conditions.

\section{Procedure}

Because of the difficulty in communicating orally with some of the deaf students, all instructions were in writing and were read by each student at the beginning of each part of the experimental sequence. The same procedure was used with the hearing and staff Ss.

For the computerspelled tasks, Ss were tested two at a time at a distance of $51 / 2 \mathrm{ft}$ from the screen. When both $S$ s were attending to the screen, the $E$ would initiate the display of letters. Each $S$ would then write out his response, taking as much time as needed. When both were again ready, the $\mathrm{E}$ would initiate another display of letters.

Because the shapes of the computerspelled letters were unfamiliar, all Ss were shown the alphabet in order for $5 \mathrm{~min}$, then 45 familiar words for practice, 15 at each of the three presentation rates, followed by 30 nonsense words, 10 at each of the three presentation rates (no 8-letter nonsense words were used). For the familiar words the Ss were instructed to guess if necessary and to leave a blank if they found it impossible to make out the word. For the nonsense words they were instructed not to guess and to make a dash for letters they missed. The sequence of stimuli was identical for each pair of Ss tested. After these practice trials, 108 familiar words were presented and, following a 5-min break, 72 nonsense words.

The same basic instructions were used for the fingerspelling conditions.

The familiar words were scored as the percent correctly guessed. Errors were classified as to mistakes and omissions. The nonsense words were scored in two ways: the number of letters correctly reported in the correct positions and the number of letters correctly reported without penalty for incorrect positioning.

\section{RESULTS \\ Correlations Across Tasks}

In general, among the deaf Ss, significant positive correlations were found among similar conditions. Thus, performance on fingerspelled words at $485 \mathrm{msec}$ is correlated $r=+.71 \quad(p<.01)$ with computer-spelled words at the slow speed (300 msec). For fingerspelled vs computerspelled nonsense words, the correlation is $r=+.55 \quad(p<.01)$. For fingerspelled words and fingerspelled nonsense words, the correlation is $\mathrm{r}=+.54$ for the proper order scoring and +.68 $(p<.01$ in each case) for the any-order scoring. These correlations drop, usually to insignificant levels, when comparisons are made across dissimilar conditions, such as a fast vs a slow rate, or across the two scoring procedures for the nonsense words. Doing well at one speed or with one method of scoring does not allow prediction to another.

The same conclusion can be drawn for the hearing $\mathrm{Ss}$ on the computerspelled conditions and the staff $S$ s on the fingerspelled conditions. Correlations between familiar and nonsense words under similar scoring and rates range from
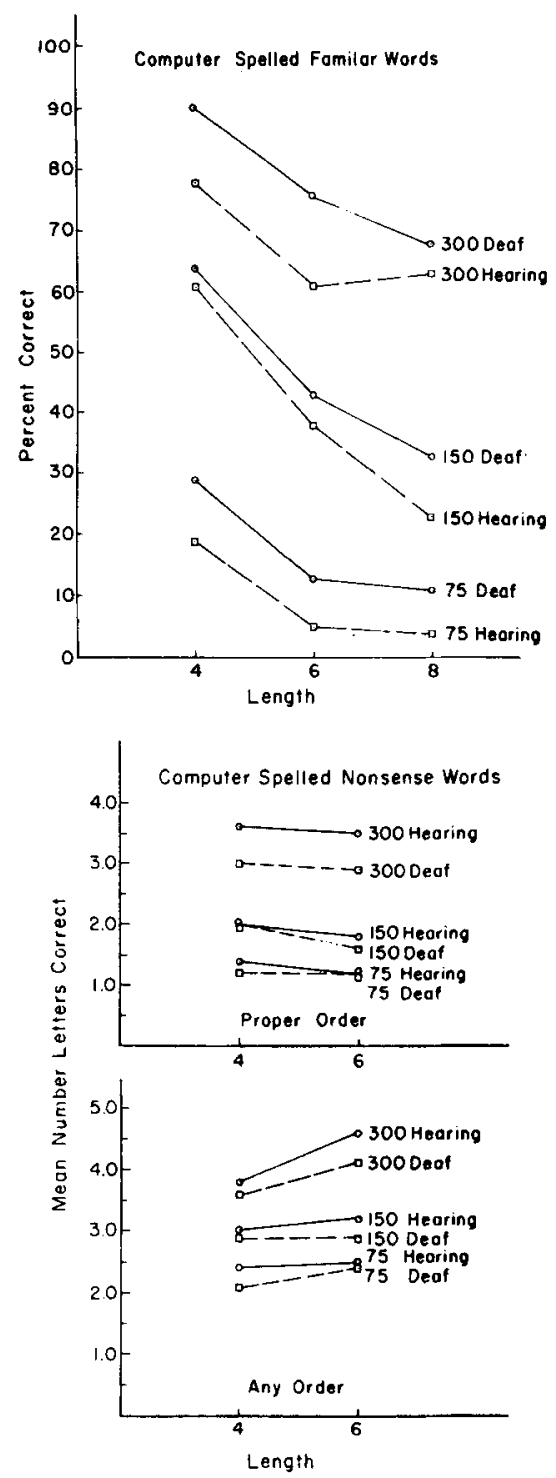

Fig. 4. Results of computerspelling of familiar and nonsense words as a function of rate of presentation, word length, and scoring criterion for deaf $S s(N=33)$ and hearing Ss $(N=19)$.

+.61 to $+.86 \quad(p<.01)$ and fall off somewhat across dissimilar scoring and rates.

Thus, while rate seems to interact with performance (as will be shown below), performance on one type of sequential task is related to that on the other one, regardless of whether the stimuli presented are familiar words or nonsense words. No significant correlations were found between any fingerspelling performance measure of the deaf Ss and reading ability, verbal ability, speechreading, or vocabulary.

Mean Differences Between Groups

Figure 4 presents the mean percent 

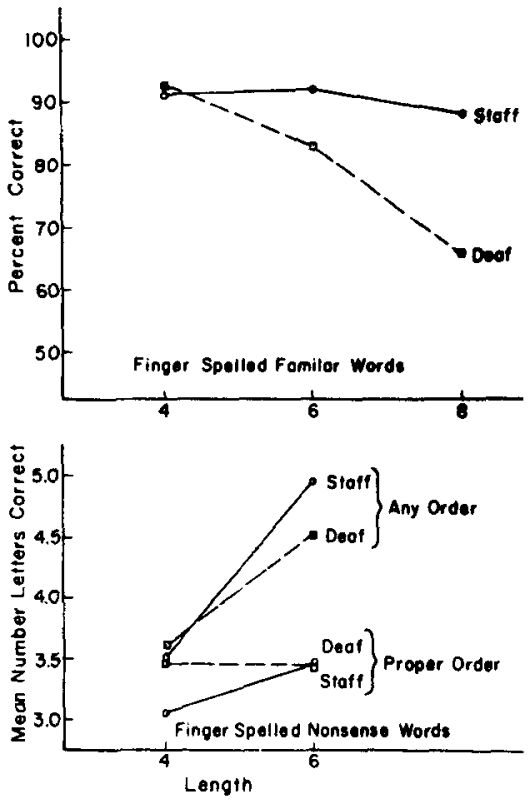

Fig. 5. Results of fingerspelling of familiar and nonsense words, as a function of word length and scoring criterion for deaf $S s(N=31)$ and experienced staff $S s$ $(\mathrm{N}=12)$.

correct for computerspelled familiar words and the mean number correct for the nonsense words, scored for proper order and any order. While hearing Ss are significantly better on the nonsense words $(\mathrm{p}<.05)$, the deaf Ss are somewhat better on familiar words $(p<.10)$. For the errors made on familiar words, deaf $S$ s were less likely to make mistakes $(\mathrm{p}<.10)$, while their omissions were equivalent.

Figure 5 presents the mean percent correct for fingerspelled familiar and nonsense words for the deaf and staff groups. The major effect is the superiority of the highly experienced staff on longer familiar words (interaction of Length by Group, $p<.05$ ). This suggests that experienced fingerspellers are attending more to the entire word, and not just to its letters. This is supported by the lack of any difference or interaction between the two groups of Ss on nonsense words.

\section{Other Variables}

Length of words. In general, longer familiar words are recognized more poorly than shorter ones. When performance drops with length, $S$ is more likely to make an omission than a mistake-apparently he is faced with too many alternatives to guess. Length also interacts with speed, as is shown in Fig. 4 for familiar words-performance drops more steeply over length for the 150-msec rate than for others. It is likely that the drop would have even been steeper for the 75 -msec rate had it not already been at the bottom, suggesting that word length is most critical when little time per letter is available. This same effect is shown for nonsense words in which the Length by Speed interaction is significant $(\mathrm{p}<.01)$ for both scoring systems. The effect appears to be a greater loss in performance on longer words at faster rates only. The length variable is also present in fingerspelled words but only for inexperienced deaf $\mathrm{Ss}$, as mentioned above.

Rate of presentation. Rate is highly significant as is seen in Fig. 4 and it interacts with length, as discussed above.

Imagery. All Ss score about $10 \%$ better on high-as compared to low-imagery words $(\mathrm{p}<.01)$. This advantage is less, however, as word length and rate increase $(p<.05$ in each case).

Rate of responding. During the fingerspelled nonsense-word conditions, it was observed that seven deaf $S$ s chose to write while watching the letters being fingerspelled. They were permitted to do this for half of the list. For the remaining half they were asked to wait until the letters of each word were sent before writing. The results of these two ways of responding are shown in Fig. 6.

Allowing Ss to write letters as they are being sent improves overall performance. It is a simpler task, requiring the processing of only one item at a time. The increase in performance is pronounced when writing the letters in proper order is required and is most pronounced for the longer (six-letter) group. Statistical tests indicate that these differences are significant $(\mathrm{p}<.05)$ for all but the four-letter group written in any order.

\section{DISCUSSION}

It is not possible to compare mean performance of computerspelling with fingerspelling directly. The latter were presented at only one rate, one nearly half the speed of the slowest computerspelled words. Further, the computerspelled letters had an atypically shaped alphabet for which Ss were not given more than a few minutes practice. Even so, comparing the 300-rnsec rate for computerspelled words with the $485-\mathrm{msec}$ rate for fingerspelled words, mean performance for the deaf $\mathrm{Ss}$ is virtually the same for four- and six-letter familiar words (about $90 \%$ correct) and they are somewhat better on eight-letter computerspelled vs fingerspelled words. Thus, while the two tasks differ greatly in familiarity, the more novel task does not overwhelm the Ss.

The correlation between finger- and computerspelled words, which reflects relative rather than absolute performance across the two tasks, also suggests some similarities between them. Further work

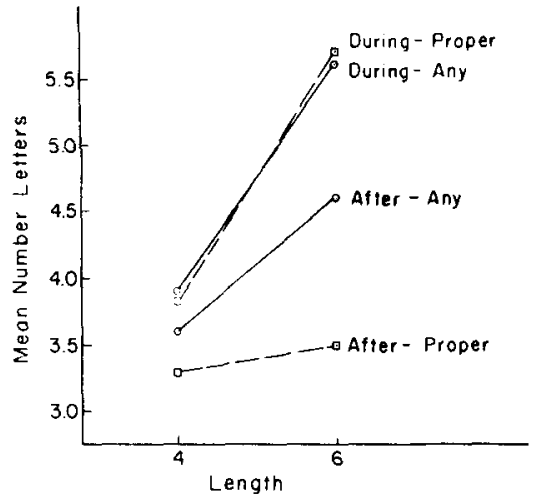

Fig. 6. Results of seven deaf Ss on computerspelled nonsense words. They first wrote their answers letter by letter as they saw them and then wrote them after the last letter appeared, as a function of word length and scoring criterion.

along this line might even be useful to determine if performance on a computerspelled task might be a prediction of ultimate achievement in learning fingerspelling-a needed screening device for schools teaching fingerspelling.

The data from this study are also relevant to the question of whether Ss identify each letter and then extract the name or meaning or the word or whether they concentrate on the word itself rather than the individual letters. Evidence here comes from two sources-difference between familiar and nonsense words and the interactions of type of word with type of $\mathrm{S}$.

While significant correlations were found between the ability to process familiar and nonsense words, both among deaf and hearing Ss, further evidence suggests that these are quite different tasks. Letters that make a fimiliar word seem to be seen as a word while letters that spell nonsense are seen only as letters. Persons experienced in attending to words when letters are sent sequentially do better at identifying words than at identifying letters. The inferior performance of the hearing $S s$ on identifying words and their superior performance on identifying letters supports this. Hearing Ss attend to each letter, regardless of whether the task is to name words or to name letters. Kolers and Katzman (1966) found that their Ss did better at naming letters than naming words. The results of this study agree with their finding: hearing $S_{s}$ do better at identifying letters. Deaf Ss, however, do better at identifying words. This situation emphasizes that the two tasks are different and that experience in the perception of sequential stimuli is important. Further, highly experienced hearing and deaf (staff) Ss were able to identify fingerspelled words 
sent at a rate of $162 \mathrm{msec}$ per letter with over $90 \%$ correctness, a rate that is over twice that which Kolers and Katzman found necessary (375 msec per letter) for the naming of words. The relative difficulty of performance on a sequential task is in part dependent upon the experience a person has with such a task. Further support comes from the finding that the staff Ss do as well with eight-letter words as with four-, suggesting it is the words being processed, not the letters. In addition, it was observed, though not measured precisely, that the staff Ss began writing their responses immediately, regardless of word length, while the deaf Ss had appreciably longer latencies, especially for the longer words.

In reading fingerspelled words a highly experienced reader is not attending to the individual letters, but rather the total pattern of the finger configuration, or at least enough of that pattern to identify the word. Fingerspelling teachers recognize that those persons who attempt to form a word by identifying each letter of a word never gain proficiency in reading fingerspelling. Further evidence that some Ss were attending to the word and not the letters that make up the word could not write it because they did not know how to spell it. This was surprising since it had just been spelled. In one case an $S$ wrote the word "bug" because he did not know how to spell the word "mosquito," which had just been sent. Koler's and Katzman's statement (1966, p. 91) that "Correct spelling does not by itself insure correct naming of the word" can be varied to read that correct recognition of the word does not by itself insure its correct spelling.

This study was not designed to test this idea explicitly, and hence these results can only be suggestive of the answer hinted at here.

\section{REFERENCES}

BORNSTEIN, H. Reading the manual alphabet. Gallaudet Research Publication, Centennial Series 2. Washington, D.C: Gallaudet College Press, 1965.

HABER, R. N., \& Nathanson, L. S. Processing of sequentially presented letters. Perception \& Psychophysics, 1969, 5, 359-361.

KOLERS, P. A., \& KATZMAN, M. T. Naming sequentially presented letters and words. Language \& Speech, 1966, 9, 84-95.

PAIVIO, A., YUILLE, J. C., \& MADIGAN, S. A. Concreteness, imagery and meaningfulness values for 425 nouns. Journal of Experimental Psychology, 1968, 76, Monograph Supplement, Part 2.

(Accepted for publication April 24, 1970.) 